\title{
Respostas fisiológicas durante a caminhada na esteira em ritmo autosselecionado: comparação entre os gêneros
}

\author{
Physiological responses during treadmill walking \\ at a self-selected pace: comparison between genders
}

\author{
Kleverton Krinski ${ }^{1}$ \\ Sergio Gregorio Dasilva \\ Hassan Mohamed Elsangedy 1 \\ Heriberto Colombo ${ }^{1,3}$ \\ Cosme F. Buzzachera \\ Bruno V. Santos ${ }^{1}$ \\ Ricardo W. Coelho \\ Wagner De Campos \\ Carlo Baldari
}

1Universidade Federal do Paraná, Centro de Pesquisa em Exercício e Esporte, Departamento de Educação Física, Curitiba, PR. Brasil.

2University of Rome, Department of Health Sciences, Itália.

3 Centro Universitário Filadelfia. Londrina, PR. Brasil.

Recebido em 05/05/08 Revisado em 19/05/08 Aprovado em 05/11/08
Resumo - O objetivo do presente estudo foi comparar o $\dot{V} \mathrm{O}_{2 \text { Máx }}, \mathrm{FC}_{\text {Máx }}, \% \mathrm{FCR}, \% \dot{V} \mathrm{O}_{2} \mathrm{R}$ e METs entre os gêneros durante a caminhada em ritmo autosselecionado e verificar se a intensidade selecionada apresenta-se fisiologicamente efetiva para manutenção ou melhora da aptidão cardiorrespiratória (ACR). Participaram 17 homens e 17 mulheres com média de idade de 23,32 \pm 3,06 anos, submetidos: (I) avaliação antropométrica e teste incremental máximo em esteira, (II) um teste de 20-minutos de caminhada em ritmo autosselecionado. $\mathrm{Na}$ análise estatística, empregou-se um teste $\mathrm{t}$ Student para medidas independentes, adotando $\mathrm{p}<0,05$. Nenhuma diferença significativa entre os gêneros para o $\% \mathrm{FC}_{\text {Máx }}(58,38 \pm 8,86$ para homens e $62,12 \pm 5,91$ para mulheres), $\% V_{2 \text { Máx }}$ (37,54 $\pm 10,75$ para homens e 40,34 $\pm 7,27$ para mulheres), \%FCR $(36,83 \pm 11,77$ para homens e $38,46 \pm 8,33$ para mulheres $)$ e $\% \dot{V}$ $\mathrm{O}_{2} \mathrm{R}(31,88 \pm 11,17$ para homens e 34,70 \pm 7,74 para mulheres). Entretanto, verifica-se que a velocidade de caminhada $\left(\mathrm{km} \cdot \mathrm{h}^{-1}\right)$ selecionada foi superior no grupo masculino $(5,96 \pm$ $0,66 ; \mathrm{p}<0,001)$ comparado ao feminino $(5,41 \pm 0,46)$, o que, possivelmente, propiciou que os METs fossem maiores nos homens $(6,07 \pm 1,57, \mathrm{p}<0,05)$ comparado as mulheres $(5,23 \pm 0,77)$. Conclui-se que ambos os gêneros autosselecionam um ritmo de caminhada considerado não efetivo para manutenção e melhora da ACR, contudo, os valores de METs demonstram-se dentro do indicado para manutenção e redução do peso corporal. As diferenças encontradas entre os gêneros para o METs podem ser decorrentes das diferenças apresentadas para a velocidade de caminhada e características antropométricas.

Palavras-chave: Exercício físico; Caminhada; Gênero.

Abstract - The objective of this study was to compare $\% \dot{V} \mathrm{O}_{2 \mathrm{Max}}, \% \mathrm{HR}_{\mathrm{Max}}, \% \mathrm{HRR}, \% \dot{V} \mathrm{O}_{2} \mathrm{R}$, and MET between genders during walking at a self-selected pace and to determine whether the self-selected pace was physiologically effective in maintaining and improving cardiorespiratory fitness. Seventeen men (age: $24.05 \pm 3.3$ years) and 17 women (age> $22.58 \pm 2.67$ years) were submitted to two experimental sessions: (I) anthropometric assessment and incremental exhaustion test, and (II) 20-min treadmill walking bouts at a self-selected pace. The independent Student t-test was used to determine differences between genders, at an alpha level of 0.05. No significant differences in $\% \mathrm{HR}_{\mathrm{Max}}(58.38 \pm 8.86$ for men and $62.12 \pm 5.91$ for women), $\% \dot{V}$ $\mathrm{O}_{2 \mathrm{Max}}$ (37.54 \pm 10.75 for men and $40.34 \pm 7.27$ for women $), \% H R R(36.83 \pm 11.77$ for men and $38.46 \pm 8.33$ for women), or $\% \dot{V} \mathrm{O}_{2} R(31.88 \pm 11.17$ for men and $34.70 \pm 7.74$ for women) were observed between genders. However, the walking speed selected $\left(\mathrm{km} . \mathrm{h}^{-1}\right)$ was higher in men $(5.96$ $\pm 0.66, p<0.001)$ than women, a finding that probably resulted in higher MET values for men $(6.07 \pm 1.57, p<0.05)$ compared to women $(5.23 \pm 0.77)$. In conclusion, both genders selected a walking pace that was not effective in maintaining or improving cardiorespiratory fitness, but MET values were within the range indicated for the maintenance and reduction of body weight. The differences in METs observed between genders might be due to differences in walking speed and anthropometric characteristics.

Key words: Physical exercise; Walking; Gender. 


\section{INTRODUÇÃO}

A caminhada é comumente utilizada por indivíduos sedentários e fisicamente ativos que desejam melhorar sua saúde e o condicionamento físico, devido a seu fácil acesso, baixo custo e risco reduzido de lesões ${ }^{1}$. Embora seja uma forma de exercício recomendada, o Colégio Americano de Medicina do Esporte (ACSM) ${ }^{1}$ tem preconizado uma intensidade entre 40/50-85\% do consumo de oxigênio de reserva $\left(\dot{V} \mathrm{O}_{2} \mathrm{R}\right)$ e/ou da frequência cardíaca de reserva (FCR) para a manutenção ou melhoria da aptidão cardiorrespiratória (ACR), a qual pode ser utilizada como um indicador de saúde geral do indivíduo. Apesar da importância de um adequado estímulo fisiológico durante a realização do exercício físico seja indiscutível para a ocorrência de adaptação orgânicas benéficas, Cox et al. ${ }^{2}$ observaram que a maioria dos sujeitos buscam se exercitar em uma intensidade autosselecionada em detrimento a uma intensidade prescrita.

Os estudos ${ }^{3.5}$ que avaliaram as respostas fisiológicas da caminhada em ritmo autosselecionado têm apresentado uma ampla variabilidade e resultados controversos, verificando estímulos considerados inadequados ${ }^{6-8}$, bem como fisiologicamente apropria$\operatorname{dos}^{3-6}$ para melhoria e manutenção da $\mathrm{ACR}^{1}$. Até o presente momento, a maioria das investigações avaliando as respostas fisiológicas durante a caminhada em intensidade autosselecionada foram realizadas em mulheres ${ }^{9,10}$, negligenciando a possivel influência do gênero. Além disso, os estudos que avaliaram a influência do gênero nas respostas fisiológicas geradas durante o exercício utilizaram atividades como, corrida e exercício em ciclo-ergômetro em intensidades pré-estabelecidas $^{11}$, e mensuraram as respostas do consumo de oxigênio $\left(\dot{V} \mathrm{O}_{2}\right)$ de maneira indireta ${ }^{3}$, fatores que limitam a comparação com as respostas fisiológicas obtidas durante a caminhada em ritmo autosselecionado. Outro aspecto que deve ser considerado é que grande parte dos estudos que investigaram as respostas fisiológicas durante a caminhada em intensidade autosselecionada basearam-se nos parâmetros referentes ao máximo consumo de oxigênio $\left(\dot{V} \mathrm{O}_{2 \text { Max }}\right)$ e frequência cardíaca máxima $\left(\mathrm{FC}_{\text {Máx }}\right)^{3-8}$, contudo, o $\mathrm{ACSM}^{1}$ tem preconizado a utilização do $\% \dot{V} \mathrm{O}_{2} \mathrm{R}$ ao invés do $\% \dot{V} \mathrm{O}_{2 \text { Máx }}$ para a precisão da intensidade do exercício, devido a disparidade de 5 a $15 \%$ entre $\%$ FCR e $\% \dot{V} \mathrm{O}_{2 \text { Máx }}$, os quais apresentam maiores variações em baixas intensidades e entre indivíduos menos condicionados e mais condicionados, fatores que se tornam reduzidos quando utilizado o \%FCR e $\% \dot{V} \mathrm{O}_{2} \mathrm{R}$ na prescrição do exercício físico ${ }^{1}$.
Desta forma, o presente estudo teve como objetivo comparar os parâmetros fisiológicos $\dot{V} \mathrm{O}_{2 \text { Máx }}$, $\mathrm{FC}_{\text {Máx }} \% \mathrm{FCR}, \% \dot{V} \mathrm{O}_{2} \mathrm{R}, \mathrm{MET}$ s entre os gêneros durante a caminhada em ritmo autosselecionado e verificar se a intensidade selecionada apresentase fisiologicamente efetiva para manutenção ou melhora da aptidão cardiorrespiratória (ACR).

\section{PROCEDIMENTOS METODOLÓGICOS}

\section{Sujeitos}

A amostra foi composta de 34 indivíduos adultos (17 homens e 17 mulheres), classificados como ativos, mediante aplicação do Questionário Internacional de Atividade Física (IPAQ) na versão curta ${ }^{12}$. As mulheres foram submetidas a uma avaliação médica inicial no intuito de excluir as que apresentassem o quadro de gravidez, alem disso, ambos os gêneros foram avaliados em relação à possível existência de problemas metabólicos, cardiovasculares, osteomioarticulares ou quaisquer outros que limitassem a prática de exercícios programados.

Para determinar o número de sujeitos, foi utilizado um cálculo amostral adotando um poder de 0,80 , alfa de 0,05 e magnitude de efeito de 1,17 , estimando 17 sujeitos para cada grupo experimental, no modelo que verifica as diferenças nas variáveis analisadas entre os dois grupos (amostras independentes), sendo suficiente para testar as hipóteses a um alfa de 0,05. O presente estudo foi aprovado pelo comitê de Ética da Universidade Federal do Paraná, conforme resolução 196/96.

\section{Sessão de familiarização}

Foi realizada uma avaliação antropométrica (peso, estatura e dobras cutâneas), e posteriormente foram esclarecidos os procedimentos e efetuada a mensuração do consumo de oxigênio de repouso $\left(\dot{V} \mathrm{O}_{2 \mathrm{Rep}}\right)$ e da frequência cardíaca de repouso $\left(\mathrm{FC}_{\mathrm{Rep}}\right)$. Após a mensuração das respostas fisiológicas de repouso, as instruções para o ajuste da velocidade através de sensores acoplados à esteira, foram explanadas, e em seguida, testadas pelos participantes na execução de 20 minutos de caminhada, associada a uma familiarização com os aparatos do analisador de gases (Cosmed K4b², Roma, Itália).

Teste incremental até a exaustão em esteira Após um intervalo de 48 horas em relação à sessão de familiarização, todos os participantes realizaram um aquecimento em esteira (marca Reebok Fitness, modelo X-fit 7, Londres, Reino Unido) de 5 minutos, a uma velocidade padrão de $4,0 \mathrm{~km} \cdot \mathrm{h}^{-1}$ 
sem inclinação. Posteriormente, um teste de esteira incremental foi conduzido mediante utilização do protocolo de Bruce ${ }^{13}$.

\section{Teste de caminhada em esteira de 20 minutos}

O teste de caminhada em esteira de 20 minutos em ritmo autosselecionado foi conduzido após aquecimento inicial de 5 minutos em velocidade de $4,0 \mathrm{~km} \cdot \mathrm{h}^{-1}$ sem inclinação. Os participantes foram instruídos a ajustar um ritmo autosselecionado, conforme os procedimentos propostos por Dishman ${ }^{4}$, mediante a utilização de sensores de controle de velocidade acoplados à esteira. Este ajuste foi realizado ad libitum durante o primeiro minuto de caminhada e posteriormente, somente nos minutos 00:05, 00:10 e 00:15. Contudo, o marcador de velocidade foi ocultado para que o avaliado não fosse influenciado pela velocidade apresentada no painel da esteira de acordo com a metodologia de Pintar et al.?

\section{Instrumentos e Procedimentos}

As variáveis antropométricas massa corporal (MC, em kg.; balança marca Toledo, modelo 2096), estatura (EST, em cm; estadiômetro marca Sanny, modelo Standard) e índice de massa corporal (IMC, em $\mathrm{kg} / \mathrm{m}^{2}$ ) foram obtidas conforme os procedimentos propostos por Lohman et al. ${ }^{14}$. A densidade corporal foi mensurada através do método de espessura de dobras cutâneas (bicipital, tricipital, subescapular e supra-ilíaca) obtidas por um único avaliador, de acordo com a equação proposta por Durnin e Womersley ${ }^{15}$. Posteriormente, o percentual de gordura corporal (\% gordura) foi obtido mediante utilização da equação de Siri ${ }^{16}$.

$\mathrm{O} \dot{V} \mathrm{O}_{2}$ foi registrado através de um sistema portátil de espirometria computadorizado de circuito aberto (marca Cosmed $\mathrm{K} 4 b^{2}$, Roma, Itália). Antes de cada teste, os sistemas de análise do $\mathrm{O}_{2}$ e $\mathrm{CO}_{2}$ foram calibrados, usando o ar ambiente e um gás com concentrações conhecidas de $\mathrm{O}_{2}$ e $\mathrm{CO}_{2}$, enquanto a turbina bidirecional (medidor de fluxo) foi calibrada usando uma seringa de 3-L (marca Hans Rudolph, modelo 5530, Kansas City, Missouri, EUA). Os dados dos testes foram obtidos respiração a respiração, e posteriormente, reduzidos a médias com intervalos de 15 segundos.

Para o cálculo do $\dot{V} \mathrm{O}_{2 \text { Rep, }}$ foram utilizados os valores médios do $\dot{V} \mathrm{O}_{2}$ obtidos nos 30 segundos finais dos 2 últimos minutos do estágio de repouso, com os participantes na posição sentada ${ }^{1} \mathrm{O} \dot{V} \mathrm{O}_{2 M a ̊ x}$ e o consumo de oxigênio no limiar ventilatório $(V$ $\mathrm{O}_{2 \mathrm{LV}}$ ) foram determinados como o maior $\dot{V} \mathrm{O}_{2}$ médio (intervalo de 1 minuto) verificado no último estágio completo do teste de esteira incremental e no limiar ventilatório, respectivamente ${ }^{17}$. A determinação do $\dot{V} \mathrm{O}_{2 \text { áx }}$ baseou-se em dois dos seguintes critérios: (a) platô do $\dot{V} \mathrm{O}_{2}$, indicado por uma diferença de $<$ 2,1 $\mathrm{mL} \cdot \mathrm{Kg}^{-1} \cdot \mathrm{min}^{-1}$ entre os dois últimos estágios do teste; (b) taxas de troca respiratória (RER) > 1,10; e (c) frequência cardíaca dentro de $\pm 5 \mathrm{bpm}$ para cada sujeito através do valor máximo predito pela idade $^{18}$. O limiar ventilatório (LV) foi calculado individualmente conforme os procedimentos estabelecidos por Caiozzo et al. ${ }^{18}$, e visualmente identificado como o ponto em que a plotagem da razão ventilação minuto por consumo de oxigênio (VE/ $\mathrm{VO}_{2}$ ) versus a razão ventilação minuto por produção de $\mathrm{CO}_{2}\left(\mathrm{VE} / \mathrm{VCO}_{2}\right)$ desvia da normalidade.

A $\mathrm{FC}_{\text {Rep }}$ foi obtida com o sujeito na posição sentada, por um período de 5 minutos, utilizando uma fita elástica ajustada ao tórax e um transmissor de rádio ligado ao sistema de análise metabólica computadorizada (Polar Oy model Sports Tester, Kempele, Finland), utilizando os valores dos 30 segundos finais obtidos nos 2 últimos minutos. Para o cálculo do $\dot{V} \mathrm{O}_{2} \mathrm{R}$ e FCR, foram utilizados os valores médios da $\dot{V} \mathrm{O}_{2}$ e FC obtidos nos 30 segundos finais dos 2 últimos minutos do estágio de repouso com os participantes na posição sentada ${ }^{1}$.

A $\mathrm{FC}_{\text {Máx }}$ e frequência cardíaca no limiar ventilatório $\left(\mathrm{FC}_{\mathrm{LV}}\right)$ foram determinadas como a maior FC média (intervalo de 1 minuto) verificada no último estágio completo do teste de esteira incremental e no limiar ventilatório, respectivamente.

$\mathrm{O}$ percentual do $\dot{V} \mathrm{O}_{2} \mathrm{R}$ foi determinado, utilizando-se o $\dot{V} \mathrm{O}_{2}$ do estágio do exercício, valor do $\dot{V} \mathrm{O}_{2 \text { Máx }}$ e $\dot{V} \mathrm{O}_{2 \mathrm{Rep}}$ de cada sujeito obtido no teste de exaustão volitiva, mediante a aplicação da seguinte fórmula:

$$
\% \mathrm{VO}_{2} \mathrm{R}=\left[\left(\mathrm{VO}_{2 \text { Estagio }}-\mathrm{VO}_{2 \mathrm{Rep}}\right) /\left(\mathrm{VO}_{\text {Máx }}-\mathrm{VO}_{2 \mathrm{Rep}}\right)\right] \times 100 .
$$

O percentual da FCR foi determinado, utilizando-se a $\mathrm{FC}$ do estágio do exercício, valor da $\mathrm{FC}_{\text {Máx }}$ e $\mathrm{FC}_{\mathrm{Rep}}$ de cada sujeito obtido no teste de exaustão volitiva, seguido da aplicação da seguinte fórmula:

$\% F C R=[($ FCEstágio - FCRep)/(FCMáx - FCRep) $]$ x 100

\section{Procedimentos estatísticos}

Medidas de tendência central e variabilidade foram utilizadas. Para testar a normalidade dos dados, utilizou-se o teste de Kolmogorov-Smirnov. As diferenças entre os gêneros para as variáveis fisiológicas foram determinadas pelo teste $\mathrm{t}$ Student para 
amostras independentes, adotando um nível de significância de $\mathrm{p}<0,05$. A magnitude de efeito (d) foi reportada para cada comparação determinada através dos procedimentos propostos por Cohen's ${ }^{19}$. Os procedimentos estatísticos foram realizados mediante a utilização do Statistical Package for the Social Sciences (SPSS, versão 13.0) for Windows.

\section{RESULTADOS}

As características dos sujeitos são apresentadas na Tabela 1, demonstrando uma MC e estatura superior nos homens.

Os parâmetros fisiológicos obtidos durante o teste incremental até a exaustão estão apresentados na Tabela 2. Segundo os critérios de classificação de
ACR propostos pelo ACSM ${ }^{1}$, a amostra apresentou um bom nível de condicionamento devido aos valores médios de $\mathrm{VO}_{2 \text { Máx }}$ estarem acima do percentil 90 para homens $\left(57,38 \mathrm{~mL} \cdot \mathrm{Kg}^{-1} \cdot \mathrm{min}^{-1}\right)$ e acima do percentil 80 para mulheres $\left(45,95 \mathrm{~mL} \cdot \mathrm{Kg}^{-1} \cdot \mathrm{min}^{-1}\right)$ classificados dentro da faixa de idade de 20 a 29 anos.

$O$ teste $t$ para amostras independentes demonstrou diferenças significativas entre os gêneros durante o teste incremental até a exaustão, nos parâmetros fisiológicos FCR, $\dot{V} \mathrm{O}_{2 \text { Máx }}, \dot{V} \mathrm{O}_{2 \mathrm{LV}}, \dot{V}$ $\mathrm{O}_{2} \mathrm{R}$ e $V E_{\text {Máx }}$, revelando que os homens possuem um maior nível de condicionamento cardiorrespiratório comparado com as mulheres.

Os parâmetros fisiológicos obtidos durante o teste de 20 minutos de caminhada em ritmo autosselecionado estão apresentados na Tabela 3.

Tabela 1. Características descritivas dos participantes.

\begin{tabular}{lcccccccc}
\hline & \multicolumn{2}{c}{ Geral } & \multicolumn{2}{c}{ Homens } & \multicolumn{2}{c}{ Mulheres } & p & $\mathrm{d}$ \\
& Média & DP & Média & DP & Média & DP & & \\
\hline Idade & 23,32 & 3,06 & 24,05 & 3,32 & 22,58 & 2,67 & 0,16 & 0,50 \\
MC & 65,39 & 10,75 & 71,96 & 10,15 & 58,81 & 6,59 & $0,00^{*}$ & 1,58 \\
Estatura & 1,68 & 0,09 & 1,75 & 0,06 & 1,62 & 0,06 & $0,00^{*}$ & 2,23 \\
IMC & 22,77 & 2,10 & 23,31 & 2,21 & 22,23 & 1,89 & 0,13 & 0,54 \\
\% gordura & 19,67 & 3,57 & 18,38 & 3,20 & 20,88 & 3,55 & 0,42 & 0,76 \\
\hline
\end{tabular}

* diferença estatisticamente significativa $(p<0,05)$ entre homens e mulheres.

Tabela 2. Parâmetros fisiológicos obtidos durante o teste incremental até a exaustão.

\begin{tabular}{|c|c|c|c|c|c|c|c|c|}
\hline & \multicolumn{2}{|c|}{ Geral } & \multicolumn{2}{|c|}{ Homens } & \multicolumn{2}{|c|}{ Mulheres } & \multirow{2}{*}{$p$} & \multirow{2}{*}{$d$} \\
\hline & Média & DP & Média & DP & Média & DP & & \\
\hline $\mathrm{FC}_{\text {Máx }}$ & 190,00 & 6,19 & 189,88 & 5,45 & 190,11 & 7,03 & 0,91 & 0,04 \\
\hline $\mathrm{FC}_{\mathrm{LV}}$ & 158,68 & 15,79 & 155,41 & 15,55 & 161,94 & 15,80 & 0,23 & 0,43 \\
\hline FCR & 122,70 & 16,05 & 128,41 & 18,78 & 117,00 & 10,47 & $0,03^{*}$ & 0,77 \\
\hline$\dot{V} \mathrm{O}_{2 \text { Máx }}$ & 51,66 & 8,13 & 57,38 & 5,92 & 45,95 & 5,63 & $0,00^{*}$ & 2,04 \\
\hline$\dot{V} \mathrm{O}_{2 \mathrm{LV}}$ & 37,23 & 7,54 & 40,10 & 7,65 & 34,36 & 6,41 & $0,02^{*}$ & 0,84 \\
\hline$\dot{V} \mathrm{O}_{2} \mathrm{R}$ & 47,31 & 7,83 & 52,61 & 6,10 & 42,00 & 5,43 & $0,00^{*}$ & 1,89 \\
\hline $\mathrm{RER}_{\text {Máx }}$ & 1,13 & 0,09 & 1,13 & 0,08 & 1,13 & 0,10 & 0,98 & 0,00 \\
\hline$\dot{V} \mathrm{E}_{M a ́ x}$ & 122,05 & 29,09 & 143,12 & 26,48 & 100,95 & 10,09 & $0,00^{*}$ & 2,17 \\
\hline
\end{tabular}

* diferença estatisticamente significativa $(p<0,05)$ entre homens e mulheres.

Tabela 3. Parâmetros fisiológicos obtidos durante o teste de caminhada de 20 minutos em ritmo autosselecionado em esteira.

\begin{tabular}{|c|c|c|c|c|c|c|c|c|}
\hline & \multicolumn{2}{|c|}{ Geral } & \multicolumn{2}{|c|}{ Homens } & \multicolumn{2}{|c|}{ Mulheres } & \multirow{2}{*}{$\mathrm{p}$} & \multirow{2}{*}{$d$} \\
\hline & Média & DP & Média & DP & Média & DP & & \\
\hline FC & 114,44 & 14,79 & 110,77 & 16,55 & 118,11 & 12,20 & 0,15 & 0,52 \\
\hline$\% \mathrm{FC}_{\text {Máx }}$ & 60,25 & 7,65 & 58,38 & 8,86 & 62,12 & 5,91 & 0,15 & 0,51 \\
\hline$\% \mathrm{FC}_{\mathrm{LV}}$ & 72,84 & 11,83 & 72,27 & 14,54 & 73,41 & 8,75 & 0,78 & 0,10 \\
\hline$\% F C R$ & 37,65 & 10,07 & 36,83 & 11,77 & 38,46 & 8,33 & 0,64 & 0,16 \\
\hline$\dot{V} \mathrm{O}_{2}$ & 19,79 & 4,53 & 21,27 & 5,52 & 18,30 & 2,70 & $0,05^{*}$ & 0,70 \\
\hline$\% \dot{V} \mathrm{O}_{2 \text { Máx }}$ & 38,94 & 9,15 & 37,54 & 10,75 & 40,34 & 7,27 & 0,38 & 0,31 \\
\hline$\% \dot{V} \mathrm{O}_{2 \mathrm{LV}}$ & 54,93 & 15,01 & 55,04 & 18,31 & 54,82 & 11,39 & 0,96 & 0,01 \\
\hline$\% \dot{V} \mathrm{O}_{2} \mathrm{R}$ & 33,29 & 9,57 & 31,88 & 11,17 & 34,70 & 7,74 & 0,39 & 0,29 \\
\hline Mets & 5,65 & 1,29 & 6,07 & 1,57 & 5,23 & 0,77 & $0,05^{*}$ & 0,70 \\
\hline Vel $\left(k m \cdot h^{-1}\right)$ & 5,68 & 0,62 & 5,96 & 0,66 & 5,41 & 0,46 & $0,00^{*}$ & 1,00 \\
\hline
\end{tabular}

* diferença estatisticamente significativa $(p<0,05)$ entre homens e mulheres. 
Podem-se observar diferenças estatísticas entre os gêneros para o $\dot{V} \mathrm{O}_{2}$ relativo, Mets e velocidade $\left(\mathrm{km} \cdot \mathrm{h}^{-1}\right)$ de caminhada durante o teste de $20 \mathrm{mi}$ nutos em caminhada de ritmo autosselecionado. Em relação à efetividade fisiológica da caminhada em ritmo autosselecionado, foi verificado que ambos os gêneros, alcançaram um \% $\mathrm{FC}_{\text {Máx }}(58,38$ $\pm 8,86$ para homens e $62,12 \pm 5,91$ para mulheres, demonstrando uma variação de $47-75 \%$ ), revelando que $94,17 \%$ das mulheres e $47,06 \%$ dos homens caminharam dentro da intensidade recomendada para o $\% \mathrm{FC}_{\text {Máx }}$ correspondente a $55-90 \%{ }^{20}$. Em relação ao $\% \mathrm{VO}_{2 \text { Máx }}(37,54 \pm 10,75$ para homens e $40,34 \pm 7,27$ para mulheres variando entre $23-59 \%$ ), verificamos que $5,6 \%$ das mulheres e $17,6 \%$ dos homens alcançaram a intensidade recomendada de $55-85 \%$ do $\% \mathrm{VO}_{2 \text { Máx }}{ }^{20}$.

No entanto, para os parâmetros fisiológicos de reserva verificamos um $\%$ FCR $(36,83 \pm 11,77$ para homens e 38,46 $\pm 8,33$ para mulheres, variando de $18-56 \%)$, e um $\% \dot{V} \mathrm{O}_{2} \mathrm{R}(31,88 \pm 11,17$ para homens e 34,70 \pm 7,74 para mulheres, exibindo uma variação de $21-56 \%$ ), revelando que, de maneira similar, $11,76 \%$ dos homens e mulheres buscaram selecionar uma intensidade de caminhada dentro do recomendado para os parâmetros fisiológicos de reserva ( $\% \dot{V} \mathrm{O}_{2} \mathrm{R}$ e $\%$ FCR) referente à 40/50-85\%, para a melhora e manutenção da $\mathrm{ACR}^{1}$. Contudo, o equivalente metabólico (Mets) $(6,07 \pm 1,57$ para homens e 5,23 $\pm 0,77$ para mulheres) revelou valores mais elevados para os homens estatisticamente, e que a intensidade de caminhada que ambos os gêneros buscaram selecionar demonstra-se dentro do recomendado para manutenção e/ou redução do peso corporal ${ }^{1}$.

\section{DISCUSSÃO}

A caminhada é considerada, atualmente, uma das práticas de exercício físico mais populares, contudo, pouco é conhecido sobre as possíveis diferenças existentes entre os gêneros nas respostas fisiológicas geradas durante um ritmo autosselecionado, e se esta intensidade escolhida pelos sujeitos apresentase fisiologicamente efetiva para manutenção ou melhora da ACR. Diretrizes anteriores do ACSM ${ }^{20}$ preconizavam a prescrição da intensidade do exercício baseando-se no $\% \mathrm{VO}_{2 \text { Máx }}$, entretanto, visto a discrepância entre o \%FCR e \%VO ${ }_{2 \text { Máx }}$ para a prescrição de exercício físico realizado em baixas intensidades, e em indivíduos com diferentes níveis de condicionamento físico ${ }^{21}$, estas diretrizes foram redefinidas, preconizando a utilização dos parâ- metros fisiológicos de reserva $\left(\% \mathrm{FCR} \text { e } \% \mathrm{VO}_{2} \mathrm{R}\right)^{1}$. Apesar dessas modificações priorizando a utilização da $\% F C R$ e do $\% \mathrm{VO}_{2} \mathrm{R}$ em detrimento do $\% \mathrm{FC}_{\text {Máx }}$ e do $\% \mathrm{VO}_{2 \text { Máx }}$, muitos pesquisadores continuam seguindo as orientações propostas por diretrizes pautadas nos parâmetros fisiológico máximos.

No presente estudo, foi verificado que ambos os gêneros autosselecionaram uma intensidade de caminhada de 60,25 $\pm 7,65 \%$ (47-75\%) para $\mathrm{FC}_{\text {máx }}$ e $38,9 \pm 9,15 \%$ (23-59\%) do $\mathrm{VO}_{2 \text { Máx }}$, contrariando os resultados encontrados por Spelman et al. ${ }^{3}$, que avaliaram 29 sujeitos praticantes regulares de caminhada, 22 homens e 7 mulheres, submetidos a um protocolo de caminhada em ritmo autosselecionado, correspondente a $69,7 \pm 8,6 \%(56-89 \%)$ da $\mathrm{FC}_{\text {Máx }}$ e $51,5 \pm 11,5 \%(35-79 \%)$ do $\mathrm{VO}_{2 \text { Máx }}$, e os achados de Murtagh et al. ${ }^{5}$, que avaliaram uma amostra de 28 homens e 54 mulheres com idade média de 40,2 \pm 13,3 (21-74 anos) sedentários, que autosselecionavam uma intensidade média de 67,3 $\pm 11,6 \%$ da $\mathrm{FC}_{\text {Máx }}$ e $59,0 \pm 13,4 \%$ do $\mathrm{VO}_{2 \text { Máx. }}$. A discrepância encontrada no presente estudo para com os achados de Spelman et al..$^{3}$ e Murtagh et al..$^{5}$ pode ser reflexo da diferença no nível de condicionamento físico e variabilidade de idade da amostra.

Os valores apresentados nos parâmetros fisiológicos $\% \mathrm{FC}_{\text {Máx }}$ e $\% \mathrm{VO}_{2 \text { máx }}$ durante caminhada em ritmo autosselecionado, na presente investigação, revelam que apenas 5,6\% das mulheres e 17,6\% dos homens alcançaram a intensidade recomendada pelo $\mathrm{ACSM}^{20}$ para o $\% \mathrm{VO}_{2 \text { Máx }}$, contudo, para o \%FC $\mathrm{Fáx}, 94,17 \%$ das mulheres e $47,06 \%$ dos homens caminharam de forma efetiva, visando melhora e manutenção da ACR. Estes achados corroboram os encontrados por Swain et al. ${ }^{17}$, que após avaliar 81 homens e 81 mulheres, com idade variando entre 18-34 anos, verificaram que durante caminhada em esteira, a uma intensidade de $40 \%$ do $\mathrm{VO}_{2 \text { Máx }}$, foi encontrado um equivalente de $63 \%$ para $\mathrm{FC}_{\text {Máx }}{ }^{\prime}$ em ambos os gêneros sem diferenças estatísticas. Esses achados revelam que o $\% \mathrm{FC}_{\text {Máx }}$ em baixas intensidades de exercício apresenta valores substancialmente maiores que os verificados para o $\% \mathrm{VO}_{2 \mathrm{Máx}}$, demonstrando a não equivalência entre estes parâmetros.

Com o intuito de reduzir a discrepância observada na utilização dos parâmetros fisiológicos $\mathrm{FC}_{\text {Máx }}$ e $\mathrm{VO}_{2 \text { Máx }}$ o $\mathrm{ACSM}^{1}$ tem preconizado a utilização do $\dot{V} \mathrm{O}_{2} \mathrm{R}$ e FCR. No presente estudo, os parâmetros fisiológicos de reserva verificados indicam que homens e mulheres buscam se exercitar em uma intensidade correspondente a 37,65 $\pm 10,07 \%$ 
(18-56\%) da FCR e $33,29 \pm 9,57 \%$ (21-56\%) do $\dot{V}$ $\mathrm{O}_{2} \mathrm{R}$, destes, $11,76 \%$ dos homens e das mulheres autosselecionaram uma intensidade considerada fisiologicamente efetiva para o $\% \dot{V} \mathrm{O}_{2} \mathrm{R}$ e $\% \mathrm{FCR}$.

Considerando as informações supracitadas, denotamos que o exercício físico realizado em intensidade autosselecionada, utilizando o $\% \mathrm{FC}_{\mathrm{Max}}$ tende a superestimar a efetividade fisiológica da caminhada, neste sentido, a aplicação de parâmetros como $\% \dot{V} \mathrm{O}_{2} \mathrm{R} \mathrm{e} \% \mathrm{FCR}$ demonstram-se indicadores mais acurados, visto que estes não sofrem influência da intensidade do exercício e nível de condicionamento dos indivíduos. Resultados semelhantes foram encontrados por Lounana et al..$^{22}$ os quais verificaram que em ciclistas de elite, a intensidade do exercício correspondente ao \% $\mathrm{FC}_{\text {Máx }}$ superestimou os valores encontrados para o $\% V \mathrm{O}_{2 \mathrm{Mx}}$ ou $\% \dot{V} \mathrm{O}_{2} \mathrm{R}$, e que $\% \mathrm{FCR}$ foi equivalente ao $\% V \mathrm{O}_{2} \mathrm{R}$, mas não ao $\% \dot{V} \mathrm{O}_{2 \text { Máx }}$.

No entanto, cabe ressaltar que embora os sujeitos não tenham realizado a caminhada em ritmo autosselecionado, em intensidades fisiologicamente efetivas para manutenção e melhora da ACR, é importante salientar que os valores de equivalente metabólico (METs) estiveram dentro do recomendado (3,0 - 6,0 METs) para manutenção e melhoria do peso corporal, caracterizando a atividade como intensidade moderada ${ }^{21}$. Além disso, na comparação entre os gêneros, foram reveladas diferenças significativas nos METs $(6,07 \pm 1,57$ para homens e 5,23 $\pm 0,77$ nas mulheres). As diferenças encontradas no gasto energético entre os homens e mulheres durante a caminhada em ritmo autosselecionado podem estar relacionadas às diferenças na massa corporal, a qual é um importante fator ligado ao custo energético do exercício, principalmente, em atividades que envolvem a sustentação do peso corporal como a caminhada ${ }^{23}$.

Outro fator ligado às diferenças apresentadas no METs poderia ser resultante das diferenças observadas na estatura, a qual é diretamente relacionada às variáveis de comprimento e frequência da passada ${ }^{24}$. Cavanagh e Williams ${ }^{24}$ observaram uma variação no consumo de oxigênio (2,6 para $\left.3,4 \mathrm{~mL} \cdot \mathrm{Kg}^{-1} \cdot \mathrm{min}^{-1}\right)$ entre comprimento e frequência da passada em corredores recreacionais, a qual influenciou o gasto energético. Segundo Bobbert ${ }^{25}$, o gasto energético da caminhada é resultante da velocidade, a qual é diretamente relacionada ao comprimento e freqência da passada.

O presente estudo apresenta algumas limitações que devem ser mencionadas como a não mensuração do comprimento e frequência da passada, entretanto, embora estas variáveis não tenham sido mensuradas, verificamos uma maior velocidade de deslocamento durante a caminhada em ritmo autosselecionado para homens comparado a mulheres, fatores que associados às diferenças antropométricas, contribuíram para um maior equivalente metabólico encontrado na amostra masculina. Outro fator que deve ser levado em consideração é que a extrapolação destes achados pode demonstrar resultados diferentes quando aplicados a outras populações (sujeitos com idade superior, diferente níveis de condicionamento, pacientes em condições especiais e sujeitos obesos).

\section{CONCLUSÃO}

Os resultados encontrados neste estudo apontam para algumas conclusões teóricas e práticas. Do ponto de vista teórico, concluímos que a utilização dos parâmetros fisiológicos de reserva diminui a discrepância verificada entre os valores de FC e $\dot{V}$ $\mathrm{O}_{2}$ durante a caminhada em ritmo autoselecionado independente do gênero, nível de condicionamento e intensidade do exercício. Em relação à efetividade fisiológica da caminhada em ritmo autoselecionado, podemos observar que ambos os gêneros buscam se exercitar em uma intensidade caracterizada como inadequada para manutenção e melhoria da ACR. No entanto, os valores de METs apresentaram-se dentro do indicado para manutenção e redução do peso corporal, demonstrando valores superiores para homens comparado a mulheres, os quais podem ser decorrentes de diferenças relacionadas a massa corporal, estatura e velocidade da caminhada. Do ponto de vista prático, os profissionais envolvidos com a prescrição do exercício físico devem regular a intensidade do exercício pautados no percentual da FCR, a qual apresenta valores percentuais similares aos do $\dot{V} \mathrm{O}_{2} \mathrm{R}$, aprimorando o cálculo da intensidade do exercício, especialmente, em indivíduos com diferentes níveis de condicionamento e em atividades de baixa intensidade como a caminhada. Além de ser considerado que a caminhada em intensidade autoselecionada apresente um complemento no gasto energético, esta não se apresenta como uma atividade adequada para melhora da ACR em indivíduos condicionados $\left(>40 \mathrm{~mL} \cdot \mathrm{Kg}^{-1} \cdot \mathrm{min}^{-1}\right)$.

\section{Agradecimentos}

Os autores agradecem o apoio recebido pela COSMED Pulmonary Function Equipament da Itália pelos equipamentos cedidos que permitiram o desenvolvimento do presente estudo. 


\section{REFERÊNCIAS BIBLIOGRÁFICAS}

1. American College of Sports Medicine RM. ACSM's guidelines for exercise testing and prescription, seventh ed. Philadelphia: Lippincott Williams and Wilkins, 2006.

2. 2. Cox KL, Burke V, Gorely TJ, Beilin LJ, Puddey IB. Controlled comparison of retention and adherence in home versus center-initiated exercise interventions in women ages 40-65 yr: The SWEAT study. Prev Med 2003;26(1):1087-1094.

3. Spelman CC, Pate RR, Macera CA, Ward DS. Selfselected exercise intensity of habitual walkers. Med Sci Sports Exerc 1993;25(1):1174-1179.

4. Dishman RD, Farqyhar KP, Cureton KJ. Responses to preferred intensity of exercise in men differing in activity level. Med Sci Sports Exerc 1994;26(1):783-790.

5. Murtagh EM, Boreham CAG, Murphy MH. Speed and exercise intensity of recreational walkers. Prev Med 2002;35(1):397-400.

6. Hills AP, Byrne NM, Wearing S, Armstrong T. Validation of the intensity of walking for pleasure in obese adults. Prev Med 2006;42(1):47-50.

7. Pintar JA, Robertson RJ, Kriska AM, Nagle E, Goss FL. The influence of fitness and body weight on preferred exercise intensity. Med Sci Sports Exerc 2006;38(1):981-988.

8. Malatesta D, Simar D, Dauvilliers Y, Candau R, Saad HE, Préfaut C. Aerobic determinants of the decline in preferred walking speed in healthy, active 65- and 80-years-old. Eur J of Physiol 2004;447(1):915-921.

9. Lind E, Joens-Matre RR, Ekkekakis P. What intensity of physical activity do previously sedentary middle-aged women select? Evidence of a coherent pattern from physiological, perceptual and affective markers. Prev Med 2005;40(1):407-419.

10. Ekkekakis P, Lind E. Exercise does not feel the same when you are overweight: the impact of self-selected and imposed intensity on affect and exertion. Int $\mathrm{J}$ of Obesity 2006;30(1)652-660.

11. Green JM, Crews TR, Bosak AM, Peveler WW. Overall and differentiated ratings of perceived exertion at the respiratory compensation threshold: effects of gender and mode. Eur J Appl Physiol 2003;89(1):445-450.

12. Matsudo S, Araújo T, Matsudo V, Andrade D, Andrade E, Oliveira LC, et al. Questinário internacional de atividade física(IPAQ): estudo devalidade e reprodutibilidade no Brasil. Rev Bras Ativ Física Saúde 2001;6(1):5-18

13. Bruce RA. Exercise testing of patients with coronary artery disease. Ann Clin Res 1971;3(1):323-332.

14. Lohman TG, Roche AF, Martorell R. Anthropometric standardization reference manual. Champaign, Illinois: Human Kinetics Books, 1988.
15. Durnin JV, Womersley J. Body fat assessed from total body density and its estimation from skinfolds thickness: measurements on 481 men and women aged from 16 to 72 years. Br J Nutr 1974;32(2):77-79.

16. Siri WE. Body composition from fluid spaces and density: analysis of methods. In: Brozek J, Henschel A. editors. Techniques for measuring body composition. Washington: National Academy of Science; 1961:223-244.

17. Swain DP, Abernathy KS, Smith CS, Lee SJ, Bunn SA. Target heart rates for the development of cardiorespiratory fitness. Med. Sci. Sports Exerc 1994;26(1):112-116.

18. Caiozzo VJ, Davis JA, Ellis JF, Azus JL, Vandagriff R, Prietto CA, et al. A comparison of gas exchange indices used to detect the anaerobic threshold. J Appl Physiol 1982;53(1):1184-1189.

19. Cohen, J. A power primer. Psychol Bull 1992;112(1):155-159.

20. Haskell WL, Lee I-M, Pate RR, Powell KE, Blair SN, Franklin BA, et al. Physical Activity and Public Health: Updated Recommendation for Adults from the American College of Sports Medicine and the American Heart Association. Med Sci Sports Exerc 2007;39(8):1423-1434.

21. Lounana J, Campion F, Noakes TD, Medelli J. Relationship between \%HRmax, \%HR Reserve, \%VO$2 \mathrm{max}$, and \%V O2 Reserve in Elite Cyclists. Me. Sci Sports Exerc 2007;39(2):350-357.

22. McArdle WD, Katch FI, Katch VL. Exercise Physiology: Energy, Nutrition and Human Performance, Philadelphia: Lippincott Williams and Wilkins, 2006.

23. Cavanagh PR, Williams KR. The effect of stride length variation on oxygen uptake during distance running. Med Sci Sports Exerc 1982;14(1):30-35.

24. Bobbert, AC. Energy expenditure in level and grade walking. J Appl Physiol 1960;15(6):1015-1021.

\author{
Endereço para correspondência \\ Kleverton Krinski \\ Universidade Federal do Paraná, Centro de Pes- \\ quisa em Exercício e Esporte, Departamento de \\ Educação Física \\ Rua Coração de Maria, 92 \\ Jardim Botânico \\ 80215-370 - Curitiba, PR. Brasil. \\ E-mail: klevertonkrinski@hotmail.com
}

